M. Balcerzak, Institute of Mathematics, Łódź Technical University, al. Politechniki 11, I-2, 90-924 Łódź, Poland and Faculty of Mathematics, Łódź University, ul. Banacha 22, 90-238 Łódź, Poland. e-mail:

mbalce@krysia.uni.lodz.pl

A. Bartoszewicz, J. Rzepecka and S. Wroński, Institute of Mathematics, Łódź Technical University, al. Politechniki 11, I-2, 90-924 Łódź, Poland. e-mail: arturbar@ck-sg.p.lodz.pl and wronskis@ck-sg.p.lodz.pl

\title{
MARCZEWSKI FIELDS AND IDEALS ${ }^{\dagger}$
}

\begin{abstract}
For an $X \neq \emptyset$ and a given family $\mathcal{F} \subset \mathcal{P}(X) \backslash\{\emptyset\}$, we consider the Marczewski field $S(\mathcal{F})$ which consists of sets $A \subset X$ such that each set $U \in \mathcal{F}$ contains a set $V \in \mathcal{F}$ with $V \subset A$ or $V \cap A=\emptyset$. We also study the respective ideal $S^{0}(\mathcal{F})$. We show general properties of $S(\mathcal{F})$ and certain representation theorems. For instance we prove that the interval algebra in $[0,1)$ is a Marczewski field. We are also interested in situations where $S(\mathcal{F})=S(\tau \backslash\{\emptyset\})$ for a topology $\tau$ on $X$. We propose a general method which establishes $S(\mathcal{F})$ and $S^{0}(\mathcal{F})$ provided that $\mathcal{F}$ is the family of perfect sets with respect to $\tau$, and $\tau$ is a certain ideal topology on $\mathbb{R}$ connected with measure or category.
\end{abstract}

\section{General properties}

The notions of $(s)$-sets and $\left(s^{0}\right)$-sets are due to Marczewski [Sz]. They have been investigated by many authors. (See [Mi1], [Mi2], [BrCo] and also [Br2], [Co], [Wa].) The scheme defining $(s)$-sets and $\left(s^{0}\right)$-sets was used for more general settings in several publications (see e.g. $[\mathrm{Mo}],[\mathrm{Bre}],[\mathrm{Pa}],[\mathrm{R}],[\mathrm{BR}]$ ). We observe that this scheme turns out interesting without any essential restrictions on a generating family of sets. Namely, let $\mathcal{F}$ be a family of nonempty subsets of a given set $X$. We put

Key Words: Marczewski sets, field of sets, Baire category, Lebesgue measure, density topology.

Mathematical Reviews subject classification: 28A05, 03E75, 06E25, 54A10, 54E52

Received by the editors August 20, 2000

* The first author was partially supported by NSF Cooperative Research Grant INT9600548 and its Polish part financed by KBN.

${ }^{\dagger}$ The main results of the paper were presented during the 13th Spring Miniconference in Real Analysis in Auburn (March 5-6, 1999). 
$S(\mathcal{F})=\{A \subset X:(\forall U \in \mathcal{F})(\exists V \in \mathcal{F})(V \subset U \cap A \vee V \subset U \backslash A)\}$, $S^{0}(\mathcal{F})=\{A \subset X:(\forall U \in \mathcal{F})(\exists V \in \mathcal{F}) V \subset U \backslash A\}$,

$H(\mathcal{F})=\{A \subset X:(\forall B \subset A) B \in \mathcal{F}\}$.

Note that $H(\mathcal{F})$ is the maximal hereditary family contained in $\mathcal{F}$. In the case when $\mathcal{F}$ consists of all perfect subsets of a given Polish space, $S(\mathcal{F})$ and $S^{0}(\mathcal{F})$ are exactly the families of classical Marczewski $(s)$-sets and $\left(s^{0}\right)$-sets.

Our notation is standard. By $\mathcal{P}(X)$ we denote the power set of $X$. Throughout the paper $X \neq \emptyset$.

Proposition 1.1. Let $\mathcal{F} \subset \mathcal{P}(X) \backslash\{\emptyset\}$. Then we have

(1) $S(\mathcal{F})$ is a field of sets,

(2) $S^{0}(\mathcal{F}) \subset H(S(\mathcal{F}))$, and $S^{0}(\mathcal{F})$ is an ideal of sets,

(3) $\mathcal{F} \cap S^{0}(\mathcal{F})=\emptyset$,

(4) $\left(\forall U \in S(\mathcal{F}) \backslash S^{0}(\mathcal{F})\right)(\exists V \in \mathcal{F}) V \subset U$,

(5) $\mathcal{F} \subset S(\mathcal{F}) \Leftrightarrow(\forall U, V \in \mathcal{F})(\exists W \in \mathcal{F})(W \subset U \cap V \vee W \subset U \backslash V)$,

(6) if $\{x\} \in \mathcal{F}$ for all $x \in X$ then $S(\mathcal{F})=\mathcal{P}(X)$ and $S^{0}(\mathcal{F})=\{\emptyset\}$.

Proof. (1) From the definition of $S(\mathcal{F})$ it immediately follows that, if $A \in$ $S(\mathcal{F})$ then $X \backslash A \in S(\mathcal{F})$. Now, assume that $A, B \in S(\mathcal{F})$. Let $C \in \mathcal{F}$. If there is a $D \in \mathcal{F}$ such that either $D \subset C \cap A$ or $D \subset C \cap B$ then $D \subset C \cap(A \cup B)$. If such $D$ does not exist, there is a $D_{1} \in \mathcal{F}$ such that $D_{1} \subset C \backslash A$ and there is a $D_{2} \in \mathcal{F}$ such that $D_{2} \subset D_{1} \backslash B$. Thus $D_{2} \subset C \backslash(A \cup B)$. Hence $A \cup B \in S(\mathcal{F})$.

Similarly, we show that $S^{0}(\mathcal{F})$ is an ideal (condition $(2)$ ). The remaining statements, except for (4), can be checked directly without troubles.

(4) Suppose that there is a $U_{0} \in S(\mathcal{F}) \backslash S^{0}(\mathcal{F})$ such that $V \backslash U_{0} \neq \emptyset$ for each $V \in \mathcal{F}$. Since $U_{0} \notin S^{0}(\mathcal{F})$, there is a $V_{0} \in \mathcal{F}$ such that $W \cap U_{0} \neq \emptyset$ for each $W \in \mathcal{F}, W \subset V_{0}$. Since $U_{0} \in S(\mathcal{F})$, there is a $W_{0} \in \mathcal{F}$ such that $W_{0} \subset V_{0} \cap U_{0}$. But then $W_{0} \backslash U_{0}=\emptyset$, a contradiction.

Corollary 1.1. If $\mathcal{F} \subset \mathcal{P}(X)$ is a field of sets, then

$$
\mathcal{F} \backslash\{\emptyset\} \subset S(\mathcal{F} \backslash\{\emptyset\}) \backslash S^{0}(\mathcal{F} \backslash\{\emptyset\}) .
$$

Proof. Use Proposition 1.1 (3) and (5).

Note that the classical $(s)$-sets and $\left(s^{0}\right)$-sets form a $\sigma$-field and a $\sigma$-ideal, respectively (the proof of $\sigma$-additivity for $\left(s^{0}\right)$-sets is based on the fusion lemma; see [Sz]). Additionally, in that case $H(S(\mathcal{F}))=S^{0}(\mathcal{F})$ [Sz, 3.1]. On the other 
hand, there are families $\mathcal{F}$ for which $H\left((S(\mathcal{F})) \neq S^{0}(\mathcal{F})\right.$ [R, Cor. 1.10]. Observe that there are cases when $S(\mathcal{F})$ forms a $\sigma$-field but $S^{0}(\mathcal{F})$ is not a $\sigma$-ideal. That happens if $X$ is an infinite set and $\mathcal{F}$ stands for the family of all infinite subsets of $X$; then $S(\mathcal{F})=\mathcal{P}(X)$ and $S^{0}(\mathcal{F})$ consists of all finite subsets of $X$. In [Pa, Lemma 2] it was proved that if $\mathcal{F} \subset S(\mathcal{F})$ and $S^{0}(\mathcal{F})$ is $\sigma$-additive then $S(\mathcal{F})$ is a $\sigma$-field.

The operation $S$ can be iterated. For a family $\mathcal{F} \subset \mathcal{P}(X)$ we define $S_{0}(\mathcal{F})=\mathcal{F}$ and $S_{\alpha}(\mathcal{F})=S\left(\cup_{\gamma<\alpha} S_{\gamma}(\mathcal{F}) \backslash\{\emptyset\}\right)$ for any ordinal $\alpha>0$. Of course we may consider only $\alpha \leq 2^{2^{\kappa}}$ where $\kappa$ is the cardinality of $X$. The families $S_{\alpha}(\mathcal{F}), \alpha>0$, are fields and from Corollary 1.1 it follows that $S_{\gamma}(\mathcal{F}) \subset S_{\alpha}(\mathcal{F})$ for any ordinals $\gamma, \alpha$ with $0<\gamma<\alpha$. In our future studies, we plan to establish the maximal number of different fields that can be obtained in a sequence of type $\left\langle S_{\alpha}(\mathcal{F}): \alpha>0\right\rangle$. In the former version of the paper we claimed incorrectly that this number is 2 . The referee has observed that it is at least 3 . (See Remark 2.2.) ${ }^{1}$

We say that two families $\mathcal{F}_{1}, \mathcal{F}_{2} \subset \mathcal{P}(X) \backslash\{\emptyset\}$ are mutually coinitial if

$$
\begin{gathered}
\left(\forall U \in \mathcal{F}_{1}\right)\left(\exists V \in \mathcal{F}_{2}\right) V \subset U \\
\text { and }\left(\forall U \in \mathcal{F}_{2}\right)\left(\exists V \in \mathcal{F}_{1}\right) V \subset U .
\end{gathered}
$$

Proposition 1.2. Let $\mathcal{F}_{1}, \mathcal{F}_{2} \subset \mathcal{P}(X) \backslash\{\emptyset\}$.

(i) If $\mathcal{F}_{1}, \mathcal{F}_{2}$ are mutually coinitial then $S\left(\mathcal{F}_{1}\right)=S\left(\mathcal{F}_{2}\right)$ and $S^{0}\left(\mathcal{F}_{1}\right)=S^{0}\left(\mathcal{F}_{2}\right)$.

(ii) Assume that $\mathcal{F}_{1} \subset S\left(\mathcal{F}_{1}\right)$ and $\mathcal{F}_{2} \subset S\left(\mathcal{F}_{2}\right)$. If $S\left(\mathcal{F}_{1}\right)=S\left(\mathcal{F}_{2}\right)$ and $S^{0}\left(\mathcal{F}_{1}\right)=S^{0}\left(\mathcal{F}_{2}\right)$ then $\mathcal{F}_{1}, \mathcal{F}_{2}$ are mutually coinitial.

Proof. (i) is evident.

(ii) Let $U \in \mathcal{F}_{1}$. Then $U \notin S^{0}\left(\mathcal{F}_{1}\right)$ by Proposition 1.1(3). Hence $U \in S\left(\mathcal{F}_{1}\right) \backslash$ $S^{0}\left(\mathcal{F}_{1}\right)=S\left(\mathcal{F}_{2}\right) \backslash S^{0}\left(\mathcal{F}_{2}\right)$ and by Proposition 1.1(4) there is a $V \in \mathcal{F}_{2}$ such that $V \subset U$. Analogously, for each $U \in \mathcal{F}_{2}$ there is a $V \in \mathcal{F}_{1}$ such that $V \subset U$.

Note that an idea similar to that contained in Proposition 1.2 was used in [Mo, Th. 1, p. 23]. The referee has asked whether the converse of (i) is true. The answer is "no" which follows from Remark 2.1 in the next section.

Now, consider a field $\Sigma$ (respectively, an ideal $\mathcal{I}$ ) of subsets of $X$. We say that:

\footnotetext{
${ }^{1} \mathrm{~S}$. Wroński has recently proved that this number is exactly 3.
} 
- $\Sigma$ (respectively, $\mathcal{I}$ ) is a topological field (respectively, a topological ideal) if there is a topology $\tau$ on $X$ such that $\Sigma$ consists of all sets with $\tau$ nowhere dense boundary (respectively, $\mathcal{I}$ consists of $\tau$-nowhere dense sets). (Cf. $[\mathrm{Ku}, \S 8 . \mathrm{V}]$.) We thus write $\Sigma=\Sigma(\tau)$ and $\mathcal{I}=N W D(\tau)$.

- $\Sigma$ (respectively, $\mathcal{I}$ ) is a Marczewski field (respectively, a Marczewski ideal) if there is a family $\mathcal{F} \subset \mathcal{P}(X) \backslash\{\emptyset\}$ such that $\Sigma=S(\mathcal{F})$ (respectively, $\mathcal{I}=S^{0}(\mathcal{F})$ ).

Note that papers $[\mathrm{BET}]$ and $[\mathrm{BBC}]$ use different terminology for Marczewski fields: the authors of [BET] say that " $\mathcal{F}$ is a basis for a MarczewskiBurstin-like characterization of $\Sigma$ ", and in $[\mathrm{BBC}], \Sigma$ is called "MarczewskiBurstin representable".

An easy connection between the above notions is contained in the following

Proposition 1.3. (Cf. $[B R]$.) If $\tau$ is a topology on $X$ then $S(\tau \backslash\{\emptyset\})=\Sigma(\tau)$ and $S^{0}(\tau \backslash\{\emptyset\})=N W D(\tau)$. Consequently, every topological field (ideal) is a Marczewski field (ideal).

From Proposition 1.2(i) we derive

Proposition 1.4. If a family $\mathcal{F} \subset \mathcal{P}(X) \backslash\{\emptyset\}$ is mutually coinitial with a topological base on $X$ then the field $S(\mathcal{F})$ is topological.

In Section 2 we shall show that the interval algebra is a Marczewski field. This will imply that there are Marczewski fields that are not topological fields. A Boolean-theoretical characterization of topological fields was given in [Wr1]. Article [CJ] was devoted to extensive studies of topological ideals; the authors considered also an additional requirement stating that an ideal consists of meager sets in some topology. In Section 2 we discuss some connections between Marczewski fields, topological fields and category bases (introduced by John Morgan II, see $[\mathrm{Mo}]$ ).

The class of Marczewski fields seems to be rich. From [Bu] it follows that the Lebesgue measurable sets in $\mathbb{R}$ form a Marczewski field. (Note that paper $[\mathrm{Bu}]$ is much earlier than $[\mathrm{Sz}]$.$) Also the sets with the Baire property in \mathbb{R}$ constitute a Marczewski field [Br1], [BET]. When we started to prepare our paper, it was not even known whether there exists a non-Marczewski field of subsets of $\mathbb{R}$. Now, our knowledge is wider. Namely, the forthcoming paper $[\mathrm{BBC}]$ contains a construction of a non-Marczewski field on $\mathbb{R}$ provided $2^{\omega}=\omega_{1}$ and $2^{\omega_{1}}=\omega_{2}$. Another result of $[\mathrm{BBC}]$ states that $2^{\omega}=\omega_{1}$ and $2^{\omega_{1}}=\omega_{2}$ imply that the Borel subsets of $\mathbb{R}$ form a Marczewski field.

For any filter $\mathcal{F}$ of the algebra $\mathcal{A}=\mathcal{P}(X)$ we denote $-\mathcal{F}=\{X \backslash E: E \in \mathcal{F}\}$ and $\mathcal{A}_{\mathcal{F}}=\mathcal{F} \cup-\mathcal{F}$. 
Proposition 1.5. For any filter $\mathcal{F}$ of $\mathcal{A}$ we have $S(\mathcal{F})=\mathcal{A}_{\mathcal{F}}$ and $S^{0}(\mathcal{F})=$ $-\mathcal{F}$.

Proof. We easily check that $\mathcal{F} \subset S(\mathcal{F})$ and $-\mathcal{F} \subset S^{0}(\mathcal{F})$. Since $\mathcal{A}_{\mathcal{F}}$ is the smallest field containing $\mathcal{F}$, we have $\mathcal{A}_{\mathcal{F}} \subset S(\mathcal{F})$. To show the reverse inclusion consider a $U \in S(\mathcal{F})$. Since $X \in \mathcal{F}$, we can find a $V \in \mathcal{F}$ such that either $V \subset U$ or $V \subset X \backslash U$. Hence either $U \in \mathcal{F}$ or $X \backslash U \in \mathcal{F}$ which means that $U \in \mathcal{A}_{\mathcal{F}}$. Thus $S(\mathcal{F}) \subset \mathcal{A}_{\mathcal{F}}$. It can be similarly shown that $S^{0}(\mathcal{F}) \subset-\mathcal{F}$.

Proposition 1.6. For a set $X$ of cardinality $|X|=\kappa$ there are $2^{2^{\kappa}}$ nonisomorphic Marczewski fields on $X$ containing all singletons.

Proof. We follow the argument given in $[\mathrm{F}]$. Let $\Phi$ consist of all filters in $\mathcal{A}=\mathcal{P}(X)$ which are intersections of two free ultrafilters. Then $|\Phi|=2^{2^{\kappa}}$ and $\mathcal{A}_{\mathcal{F}_{1}} \neq \mathcal{A}_{\mathcal{F}_{2}}$ for any distinct $\mathcal{F}_{1}, \mathcal{F}_{2} \in \Phi$. Additionally, $\{x\} \in \mathcal{A}_{\mathcal{F}}$ for any $x \in X$ and $\mathcal{F} \in \Phi$. Thus, by Proposition 1.5, there are $2^{2^{\kappa}}$ Marczewski fields on $X$ containing all singletons. Any isomorphism between subalgebras of $\mathcal{P}(X)$ containing all singletons is induced by a bijection from $X$ to $X$. Hence each isomorphism class of such subalgebras has at most $2^{\kappa}$ elements. Finally, observe that if $h$ is a bijection of $X$ onto $X$ and $\mathcal{F} \in \Phi$ then

$$
\{h[U]: U \in S(\mathcal{F})\}=S(\{h[V]: V \in \mathcal{F}\}) \text { and }\{h[V]: V \in \mathcal{F}\} \in \Phi .
$$

Thus there are $2^{2^{\kappa}}$ different classes of isomorphic Marczewski fields on $X$ containing all singletons.

\section{Marczewski fields, topological fields and category bases}

Let $X=[0,1)$. The family of all finite unions of half-open intervals $[a, b)$ (where $0 \leq a<b \leq 1$ ) form a field of subsets of $X$. It is called the interval algebra of $X[\mathrm{~K}, 1.11]$.

Theorem 2.1. The interval algebra $\mathcal{A}$ of $X=[0,1)$ is a Marczewski field.

Proof. Let $\mathbb{Q}$ stand for the set of all rationals and let $\mathfrak{c}$ denote the cardinality of $\mathbb{R}$. Consider the equivalence relation $x \sim y \Longleftrightarrow x-y \in \mathbb{Q}$. Let $F:[0,1] \rightarrow$ $\mathbb{R} / \sim$ be a one-to-one function such that $x \notin F(x)$ for $x \in[0,1]$. (Note that $F$ can be easily constructed by transfinite induction. Indeed, arrange all points of $[0,1]$ into a one-to-one sequence $x_{\gamma}, \gamma<\mathfrak{c}$, and consider an $\alpha<\mathfrak{c}$. If the values $F\left(x_{\gamma}\right)$ for $\gamma<\alpha$ have been defined, we pick $x \in[0,1] \backslash \bigcup_{\gamma<\alpha}\left[F\left(x_{\gamma}\right)\right] \backslash\left[x_{\alpha}\right]$ and put $F\left(x_{\alpha}\right)=[x]$ where $[x]$ denotes the respective equivalence class.)

For $x \in[0,1]$ let

$\mathcal{F}_{r}(x)=\{([x, x+\varepsilon) \backslash F(x)) \cap X: \varepsilon>0\}$, 
$\mathcal{F}_{l}(x)=\{((x-\varepsilon, x) \backslash F(x)) \cap X: \varepsilon>0\}$, $\mathcal{F}(x)=\mathcal{F}_{l}(x) \cup \mathcal{F}_{r}(x)$.

Note that $\mathcal{F}_{r}(1)=\mathcal{F}_{l}(0)=\emptyset$, otherwise $\mathcal{F}_{r}(x)$ and $\mathcal{F}_{l}(x)$ are nonempty. Finally, let $\mathcal{F}=\bigcup_{x \in[0,1]} \mathcal{F}(x)$.

From the definitions of $\mathcal{F}$ and $S(\mathcal{F})$ it easily follows that $[a, b) \in S(\mathcal{F})$ for any $a, b$ with $0 \leq a<b \leq 1$. Since $S(\mathcal{F})$ is a field of sets, we have $\mathcal{A} \subset S(\mathcal{F})$.

Claim 1. Let $x \in[0,1]$ and $k \in\{r, l\}$. If $U \in \mathcal{F}_{k}(x)$ then for each $y \in[0,1]$ and for each $V \in \mathcal{F}(y)$ such that $V \subset U$, we have $y=x$, and moreover $V \in \mathcal{F}_{k}(x)$.

Indeed, suppose that $y \neq x$. Let $U=I \backslash F(x)$ and $V=J \backslash F(y)$ where $I$ and $J$ are the respective intervals. From $V \subset U$ and the density of $F(x)$ it follows that $\emptyset \neq J \cap F(x) \subset J \cap F(y)$ which contradicts the disjointness of $F(x)$ and $F(y)$. Thus $y=x$ and so, $V \in \mathcal{F}_{m}(x)$ for some $m \in\{r, l\}$. However, $m=k$ since otherwise $U \cap V=\emptyset$.

We have already observed that $\mathcal{A} \subset S(\mathcal{F})$. To prove that $S(\mathcal{F}) \subset \mathcal{A}$ fix an $A \in S(\mathcal{F}) \backslash\{\emptyset\}$.

Claim 2. For each $x \in A$ there exists an $\varepsilon>0$ such that $[x, x+\varepsilon) \subset A$. For each $x \in X \backslash A$ there exists an $\varepsilon>0$ such that $[x, x+\varepsilon) \cap A=\emptyset$.

The latter assertion follows from the former applied to $X \backslash A$. To show the former assertion, suppose $x \in A$ and $[x, x+\varepsilon) \backslash A \neq \emptyset$ for each $\varepsilon>0$. Consider a $U \in \mathcal{F}_{r}(x)$. Since $A \in S(\mathcal{F})$, there is a $V \in \mathcal{F}$ such that either $V \subset U \cap A$ or $V \subset U \backslash A$. By Claim 1 we have $V \in \mathcal{F}_{r}(x)$. Since $x \in A \cap V$, we infer that $V \subset U \cap A$. Let $V=([x, x+\varepsilon) \backslash F(x)) \cap X$ where $\varepsilon>0$. We may assume that $x+\varepsilon \leq 1$. By our supposition, pick a $y \in(x, x+\varepsilon) \backslash A$. Let $\tilde{V}=[y, x+\varepsilon) \backslash F(y)$. Then $\tilde{V} \in \mathcal{F}_{r}(y)$ and since $A \in S(\mathcal{F})$, there is a $W \in \mathcal{F}$ such that either $W \subset \tilde{V} \cap A$ or $W \subset \tilde{V} \backslash A$. Again, by Claim 1, we have $W \in \mathcal{F}_{r}(y)$, so we may assume that $W=\left[y, y+\varepsilon_{1}\right) \backslash F(y)$ where $y+\varepsilon_{1} \leq x+\varepsilon$. Since $y \notin A$, we have $W \cap A=\emptyset$. The set $\left[y, y+\varepsilon_{1}\right) \backslash(F(x) \cup F(y))$ is nonempty (uncountable) contained in $[x, x+\varepsilon) \backslash F(x)=V \subset A$ and simultaneously in $\left[y, y+\varepsilon_{1}\right) \backslash F(y)=W \subset X \backslash A$. Contradiction.

Claim 3. For each $x \in(0,1]$ there exists an $\varepsilon>0$ such that either $(x-\varepsilon, x) \subset$ $A$ or $(x-\varepsilon, x) \cap A=\emptyset$.

To show the claim, suppose that there exists an $x \in(0,1]$ such that $(x-$ $\varepsilon, x) \backslash A \neq \emptyset$ and $(x-\varepsilon, x) \cap A \neq \emptyset$ for each $\varepsilon>0$. Let $U \in \mathcal{F}_{l}(x)$. Since $A \in S(\mathcal{F})$, there is a $V \in \mathcal{F}$ such that either $V \subset U \cap A$ or $V \subset U \backslash A$. By Claim 1 we have $V \in \mathcal{F}_{l}(x)$ and we may assume that $V=(x-\varepsilon, x) \backslash F(x)$ 
where $x-\varepsilon \geq 0$. If $V \subset A$, by our supposition we can pick $y \in(x-\varepsilon, x) \backslash A$. By Claim 2 there is an $\varepsilon_{1}>0$ such that $\left[y, y+\varepsilon_{1}\right) \cap A=\emptyset$ and we may assume that $y+\varepsilon_{1} \leq x$. On the other hand, $\emptyset \neq\left[y, y+\varepsilon_{1}\right) \backslash F(x) \subset(x-\varepsilon, x) \backslash F(x) \subset V \subset A$, a contradiction. If $V \cap A=\emptyset$, by our supposition we can pick $y \in(x-\varepsilon, x) \cap A$. By Claim 2 there is an $\varepsilon_{1}>0$ such that $\left[y, y+\varepsilon_{1}\right) \subset A$ and we may assume that $y+\varepsilon_{1} \leq x$. On the other hand, $\emptyset \neq\left[y, y+\varepsilon_{1}\right) \backslash F(x) \subset(x-\varepsilon, x) \backslash F(x)=$ $V \subset X \backslash A$, a contradiction.

From Claim 2 it follows that each connected component $I$ of $A$ is a nondegenerate interval with $b=\sup I \notin I$. Denote $a=\inf I$ and observe that $a \in I$. Indeed, suppose that $a \notin I$. We know that $[a, b) \in S(\mathcal{F})$. Thus $A \cap[a, b)=(a, b) \in S(\mathcal{F})$ and consequently $[a, b) \backslash(a, b)=\{a\} \in S(\mathcal{F})$ which contradicts Claim 2.

From the above we infer that $A$ is a union of at most countable family of pairwise disjoint intervals of type $[a, b)$. This family however cannot be infinite. Indeed, suppose that $A=\bigcup_{n=1}^{\infty}\left[a_{n}, b_{n}\right)$ with $\left[a_{n}, b_{n}\right) \subset X, n \geq 1$, pairwise disjoint. Pick a strictly monotonic subsequence $\left(a_{k_{n}}\right)$ of $\left(a_{n}\right)$. If $a_{k_{n}} \searrow x$, we apply Claim 2 to $x$ and we obtain a contradiction. If $a_{k_{n}} \nearrow x$, we apply Claim 3 to $x$ and we obtain a contradiction.

Thus we have proved that $A \in \mathcal{A}$. Consequently, $S(\mathcal{F}) \subset \mathcal{A}$.

Remark 2.1. Observe that in the above construction, we can choose, for $i=1,2$, one-to-one functions $F^{(i)}:[0,1] \rightarrow \mathbb{R} / \sim$ with disjoint ranges, and such that $x \notin F^{(i)}(x)$ for each $x \in[0,1]$. Then $\mathcal{A}=S\left(\mathcal{F}^{(1)}\right)=S\left(\mathcal{F}^{(2)}\right)$ where $\mathcal{F}^{(i)}(i=1,2)$ is associated with $F^{(i)}$ as in the proof of Theorem 2.1. Since $H(\mathcal{A})=\{\emptyset\}$, we have $S^{0}\left(\mathcal{F}^{(1)}\right)=S^{0}\left(\mathcal{F}^{(2)}\right)=\{\emptyset\}$ by Proposition 1.1(2). However, the argument for Claim 1 shows that $\mathcal{F}^{(1)}$ and $\mathcal{F}^{(2)}$ are not mutually coinitial. Thus the converse of (i) in Proposition 1.2 is false.

Remark 2.2. Since $\mathcal{A} \backslash\{\emptyset\}$ and the family of nonempty open sets in $[0,1)$ are mutually coinitial, the field $\Sigma=S(\mathcal{A} \backslash\{\emptyset\})$ consists of all sets in $[0,1)$ with nowhere dense boundary, and $S(\Sigma \backslash\{\emptyset\})=\mathcal{P}([0,1))$ by Proposition 1.1(6). So we have 3 different fields obtained by the iteration of $S(\cdot)$.

Corollary 2.1. There exists an Marczewski field which is not a topological field.

Proof. This follows from Theorem 2.1 since every topological field has an atom $[\mathrm{Wr} 1]$ and the algebra $\mathcal{A}$ has no atoms.

Although the class of topological subfields of $\mathcal{P}(X)$ is smaller than the class of Marczewski fields, the former can be used to get the following representation result: 
Theorem 2.2. Every field $\Sigma$ of subsets of $X$ is equal to the intersection of all topological fields containing $\Sigma$.

Proof. If $\Sigma=\mathcal{P}(X)$, the assertion is obvious. Assume that $\Sigma \neq \mathcal{P}(X)$. It suffices to show that for each $A \notin \Sigma$ there is a topological field $\Sigma_{A} \supset \Sigma$ with $A \notin \Sigma \Sigma_{A}$. So, let $A \notin \Sigma$. By [Wr2, Lemma 2] we find an ultrafilter $\mathcal{F}_{A}$ of the field $\Sigma$ such that no subset of $A$ is in $\mathcal{F}_{A}$ and no subset of $X \backslash A$ is in $\mathcal{F}_{A}$. Thus $A \notin S\left(\mathcal{F}_{A}\right)$. Put $\Sigma_{A}=S\left(\mathcal{F}_{A}\right)$. Observe that $\Sigma_{A}$ is a topological field since $\mathcal{F}_{A}$ forms a topological base, and thus Proposition 1.4 can be used. Because $\mathcal{F}_{A}$ is an ultrafilter of $\Sigma$, we have $\Sigma \subset S\left(\mathcal{F}_{A}\right)$.

Recall $[\mathrm{Mo}]$ that a pair $(X, \mathcal{C})$ is said to be a category base if $\mathcal{C}$ stands for a family of subsets of a nonempty set $X$, and nonempty sets in $\mathcal{C}$, called regions, satisfy the following axioms:

$1^{0} \cup \mathcal{C}=X$

$2^{0}$ Let $A$ be a region and $\mathcal{D}$ - a nonempty family of disjoint regions with $|\mathcal{D}|<|\mathcal{C}|$. Then

- if $A \cap(\bigcup \mathcal{D})$ contains a region then there is a region $D \in \mathcal{D}$ such that $A \cap D$ contains a region;

- if $A \cap(\bigcup \mathcal{D})$ contains no region then there is a region $B \subset A \backslash \bigcup \mathcal{D}$.

A set $E \subset X$ is called singular if $E \in S^{0}(\mathcal{C} \backslash\{\emptyset\})$.

Theorem 2.3. Assume that for a family $\mathcal{F} \subset \mathcal{P}(X) \backslash\{\emptyset\}$ we have $X \in \mathcal{F} \subset$ $S(\mathcal{F})$ and $\bigcup\left(S^{0}(\mathcal{F}) \cap\{U \cap V: V \in \mathcal{F}\}\right) \in S^{0}(\mathcal{F})$ for each $U \in \mathcal{F}$. Then $(X, \mathcal{F})$ is a category base whose ideal of singular sets equals $S^{0}(\mathcal{F})$.

Proof. It is enough to check condition $2^{0}$ defining a category base. Let $F, F_{t} \in \mathcal{F}$ for $t \in T$ (where $T$ is an arbitrary set of indices). Assume that $F \cap \bigcup_{t \in T} F_{t}$ contains a set from $\mathcal{F}$. Then there exists a $t_{0} \in T$ such that $F \cap F_{t_{0}}$ contains a set from $\mathcal{F}$. Indeed, suppose that it is not the case. Since $\mathcal{F} \subset S(\mathcal{F})$, we have $F \cap F_{t} \in S(\mathcal{F})$ for each $t \in T$. Hence by Proposition 1.1(4) we get $F \cap F_{t} \in S^{0}(\mathcal{F})$. Thus, by assumption, we have $\bigcup_{t \in T}\left(F \cap F_{t}\right) \in S^{0}(\mathcal{F})$ which yields a contradiction (cf. Proposition 1.1(3)). Assume now that $F \cap \bigcup_{t \in T} F_{t}$ contains no set from $\mathcal{F}$. Then every set $F \cap F_{t}, t \in T$, contains no set from $\mathcal{F}$. As before we infer that $F \cap F_{t} \in S(\mathcal{F})$ for $t \in T$ and moreover $F \cap F_{t} \in S^{0}(\mathcal{F})$. By assumption we have $\bigcup_{t \in T}\left(F \cap F_{t}\right) \in S^{0}(\mathcal{F})$ and consequently,

$$
F \backslash \bigcup_{t \in T}\left(F \cap F_{t}\right) \in S(\mathcal{F}) \backslash S^{0}(\mathcal{F})
$$


since $F \in S(\mathcal{F}) \backslash S^{0}(\mathcal{F})$. Hence, by Proposition 1.1(4), there is a set from $\mathcal{F}$ contained in $F \backslash \bigcup_{t \in T} F_{t}$.

\section{Marczewski fields and perfect sets}

Let $(X, \tau)$ be a topological space. By a $\tau$-perfect set we mean a nonempty $\tau$-closed set without isolated points. Let $\operatorname{Perf}(\tau)$ stand for the family of all $\tau$-perfect subsets of $X$. As it was mentioned in Section 1, if $X$ is a Polish space with topology $\tau$ then $S(\operatorname{Perf}(\tau))$ and $S^{0}(\operatorname{Perf}(\tau))$ are exactly the classical families of Marczewski $(s)$-sets and $\left(s^{0}\right)$-sets. In [R], studies of $S(\operatorname{Perf}(\tau))$ and $S^{0}(\operatorname{Perf}(\tau))$ for other topological spaces were initiated. (See also [BR].) In this section we propose a general method which enables us to reprove some results of $[\mathrm{R}]$ and $[\mathrm{BR}]$, and to show new applications.

If $\tau$ is a given topology on $X$ and we want to characterize $S(\operatorname{Perf}(\tau))$, we shall use the following scheme:

$1^{0}$ we conjecture that $S(\operatorname{Perf}(\tau))=\Sigma$ where $\Sigma$ is a known field of sets,

$2^{0}$ we know that $\Sigma=S(\mathcal{F})$ for some family $\mathcal{F} \subset \mathcal{P}(X) \backslash\{\emptyset\}$;

$3^{0}$ in aim to confirm our conjecture $1^{0}$, it is enough (by Proposition 1.2(i)) to check that $\operatorname{Perf}(\tau)$ and $\mathcal{F}$ are mutually coinitial.

A similar method works for $S^{0}(\operatorname{Perf}(\tau))$. The above scheme will be illustrated by examples dealing with some "ideal topologies" on $\mathbb{R}$.

Let $\tau$ be a topology on $X$, and let $\mathcal{I} \subset \mathcal{P}(X)$ be a $\sigma$-ideal containing all singletons. The family

$$
\mathcal{B}_{\mathcal{I}}^{\star}=\{U \backslash A: U \in \tau \& A \in \mathcal{I}\}
$$

forms a base for a topology $\tau_{\mathcal{I}}^{\star}$, on $X$, stronger than $\tau$, which will be called the Hashimoto topology associated with $\tau$ and $\mathcal{I}$. If $\tau$ is second countable (or even hereditary Lindelöf) then $\tau_{\mathcal{I}}^{\star}=\mathcal{B}_{\mathcal{I}}^{\star}$. (See $[\mathrm{H}],[\mathrm{JH}]$, [LMZ].) The following property is well known.

Lemma 3.1. (Cf. [BR]). Let $\mathcal{I}$ be a $\sigma$-ideal of subsets of a separable metric space $X$ and let $\mathcal{I}$ contain all singletons. A set $F \subset X$ is $\tau_{\mathcal{I}}^{\star}$-perfect if and only if $F$ is $\tau$-perfect and $U \cap F \notin \mathcal{I}$ for each $U \in \tau$ with $U \cap F \neq \emptyset$.

By $\mathcal{M}$ and $\mathcal{N}$ we denote, respectively, the $\sigma$-ideals all meager (i.e. of the first category) sets and of all Lebesgue null sets in $\mathbb{R}$. We shall consider the Hashimoto topologies $T_{\mathcal{M}}^{\star}$ and $T_{\mathcal{N}}^{\star}$ where $T$ stands for the natural topology 
on $\mathbb{R}$. Let $\mathcal{D}_{\mathcal{N}}$ denote the density topology on $\mathbb{R}$. (See e.g. [O] or [CLO].) Wilczyński in [W1] introduced the category analogue of the density topology which will be denoted by the $\mathcal{D}_{\mathcal{M}}$. Since topology $\mathcal{D}_{M}$ is less known, let us give necessary definitions. A number $x \in \mathbb{R}$ is called a category density point of a set $A \subset \mathbb{R}$ with the Baire property if each increasing sequence $\left\{n_{k}\right\}$ of positive integers has a subsequence $\left\{n_{m_{k}}\right\}$ such that the sequence of characteristic functions

$$
\chi_{[-1,1] \cap n_{m_{k}}(A-x)}(t)
$$

(where $n_{m_{k}}(A-x)=\left\{n_{m_{k}}(a-x): a \in A\right\}$ ) tends to $\chi_{[-1,1]}(t)$ for all points $t \in \mathbb{R}$ except for those belonging to a meager set. If $[-1,1]$ is replaced by $[-1,0]$ or $[0,1]$, we get the respective notions of one-sided category density points. Topology $\mathcal{D}_{\mathcal{M}}$ consists of all sets $A \subset \mathbb{R}$ with the Baire property, such that each point of $A$ is a category density point of $A$. There are many analogies between $\mathcal{D}_{\mathcal{M}}$ and $\mathcal{D}_{\mathcal{N}}$; for details, see [PWW], [W2] and [CLO].

It is known that the only possible inclusions between the above-mentioned topologies are the following $T \subsetneq T_{\mathcal{M}}^{\star} \subsetneq \mathcal{D}_{\mathcal{M}}$ and $T \subsetneq T_{\mathcal{N}}^{\star} \subsetneq \mathcal{D}_{\mathcal{N}}$. (See [LJW].)

The following proposition is due to $\mathrm{W}$. Wilczyński (oral communication).

Proposition 3.1. Every $\mathcal{D}_{\mathcal{M}}$-perfect set has nonempty $T$-interior.

Proof. Let $F$ be a $\mathcal{D}_{\mathcal{M}}$-perfect set. Then $F$ is nonmeager. Indeed, if $F \in \mathcal{M}$ and $x \in F$ then $U=(\mathbb{R} \backslash F) \cup\{x\} \in \mathcal{D}_{\mathcal{M}}$ and $F \cap U=\{x\}$ which contradicts the fact that $F$ is a $\mathcal{D}_{\mathcal{M}}$-perfect set. Since $F$ is a nonmeager set with the Baire property, there is an open interval $V$ such that $F \cap V$ is comeager in $V$. We claim that $V \subset F$. Let $x \in V$ and let $W$ be a $\mathcal{D}_{\mathcal{M}}$-neighborhood of $x$. Thus $x$ is a category density point of the both sets $V$ and $W$. Consequently, $V \cap W \notin \mathcal{M}$. Since $F \cap V$ is comeager in $V$, we thus have $F \cap W \neq \emptyset$. Hence $x$ belongs to the the $\mathcal{D}_{\mathcal{M}}$-closure of $F$ (equal to $F$ ).

Let $\Sigma_{\mathcal{M}}$ and $\Sigma_{\mathcal{N}}$ denote, respectively, the $\sigma$-fields of all sets with the Baire property and of all Lebesgue measurable sets in $\mathbb{R}$. As in Section 1, $\Sigma(T)$ stands for the field of all subsets of $\mathbb{R}$ with nowhere dense boundary, and $N W D(T)$ - the ideal of nowhere dense subsets of $\mathbb{R}$.

Theorem 3.1. (a) $S\left(\operatorname{Perf}\left(\mathcal{D}_{\mathcal{N}}\right)\right)=\Sigma_{\mathcal{N}}, S^{0}\left(\operatorname{Perf}\left(\mathcal{D}_{\mathcal{N}}\right)\right)=\mathcal{N}$. $(\operatorname{See}[R]$.

(b) $S\left(\operatorname{Perf}\left(T_{\mathcal{N}}^{\star}\right)\right)=\Sigma_{\mathcal{N}}, S^{0}\left(\operatorname{Perf}\left(T_{\mathcal{N}}^{\star}\right)\right)=\mathcal{N} \cdot(\operatorname{See}[B R]$.)

(c) $S\left(\operatorname{Perf}\left(T_{\mathcal{M}}^{\star}\right)\right)=\Sigma(T), S^{0}\left(\operatorname{Perf}\left(T_{\mathcal{M}}^{\star}\right)\right)=N W D(T)$. (See [BR].)

(d) $S\left(\operatorname{Perf}\left(\mathcal{D}_{\mathcal{M}}\right)\right)=\Sigma(T), S^{0}\left(\operatorname{Perf}\left(\mathcal{D}_{\mathcal{M}}\right)\right)=N W D(T)$. 
Proof. (a) Let $\mathcal{F}$ denote the family of all $T$-perfect sets of positive measure. Burstin $[\mathrm{Bu}]$ proved that $\Sigma_{\mathcal{N}}=S(\mathcal{F})$. It is not hard to prove that $\mathcal{F}$ and $\operatorname{Perf}\left(\mathcal{D}_{\mathcal{N}}\right)$ are mutually coinitial (cf. [R, Lemma 3.2]). Thus by our scheme we have $S\left(\operatorname{Perf}\left(\mathcal{D}_{\mathcal{N}}\right)\right)=\Sigma_{\mathcal{N}}$. Moreover $S^{0}(\mathcal{F}) \subset H(S(\mathcal{F}))=H\left(\Sigma_{\mathcal{N}}\right)=\mathcal{N}$ and $\mathcal{N} \subset \Sigma_{\mathcal{N}}=S(\mathcal{F})$. But $\mathcal{N} \subset S(\mathcal{F})$ easily implies that $\mathcal{N} \subset S^{0}(\mathcal{F})$. Hence $\mathcal{N}=S^{0}(\mathcal{F})=S^{0}\left(\operatorname{Perf}\left(\mathcal{D}_{\mathcal{N}}\right)\right)$.

(b) If $\mathcal{F}$ is as in (a), Lemma 3.1 easily implies that $\mathcal{F}$ and $\operatorname{Perf}\left(T_{\mathcal{N}}^{\star}\right)$ are mutually coinitial (cf. $[\mathrm{BR}]$ ). The rest is the same as in (a).

(c) From Lemma 3.1 it follows that $T_{\mathcal{M}}^{\star}$-perfect sets have nonempty $T$ interior. Also, a nonempty $T$-open set contains a $T_{\mathcal{M}}^{\star}$-perfect set (a closed nondegenerate interval). Hence $\operatorname{Perf}\left(T_{\mathcal{M}}^{\star}\right)$ and $T \backslash\{\emptyset\}$ are mutually coinitial. Thus the assertion follows from Proposition 1.3.

(d) As in (c) it suffices to prove that $\operatorname{Perf}\left(\mathcal{D}_{\mathcal{M}}\right)$ and $T \backslash\{\emptyset\}$ are mutually coinitial. Firstly, by Proposition 3.1, every $\mathcal{D}_{\mathcal{M}}$-perfect set has nonempty $T$-interior. Secondly, let us show that a nondegenerate interval $[a, b]$ is a $\mathcal{D}_{\mathcal{M}}$-perfect set. Indeed, $[a, b]$ is obviously $\mathcal{D}_{\mathcal{M}}$-closed. Let $x \in[a, b]$ and $x \in U \in \mathcal{D}_{\mathcal{M}}$. Then $x$ is a category density point of $U$ and $x$ is at least a one-sided category density point of $[a, b]$. Hence $U \cap[a, b] \notin \mathcal{M}$ and so, $x$ is a $\mathcal{D}_{\mathcal{M}}$-accumulation point of $[a, b]$.

Note that statements (a) and (b),(c) of Theorem 3.1 can be extended to cases dealing with spaces more general than $\mathbb{R}$, as it was mentioned in $[R]$ and $[\mathrm{BR}]$. Topology $\mathcal{D}_{\mathcal{M}}$ can be considered in certain linear topological spaces and statement (d) of Theorem 3.1 then holds.

Assertions (a),(d) and (b),(c) of Theorem 3.1 show a kind of asymmetry between measure and category. Knowing (a),(b) we rather expected to obtain $\Sigma_{\mathcal{M}}$ and $\mathcal{M}$ as the respective Marczewski families in (c),(d). So the following problem appears:

Problem 3.1. Find a topology $\tau$ on $\mathbb{R}$ such that $\Sigma_{\mathcal{M}}$ (the $\sigma$-field of all subsets of $\mathbb{R}$ with the Baire property) is of the form $S(\operatorname{Perf}(\tau))$.

Note that Brown [Br1] (see also [BET]) showed the equalities $\Sigma_{\mathcal{M}}=S(\mathcal{G})$ and $\mathcal{M}=S^{0}(\mathcal{G})$ provided that $\mathcal{G}$ consists of sets of the form $U \backslash F$ where $U$ is open and $F$ is an $F_{\sigma}$ meager set. This easily implies that $\Sigma_{\mathcal{M}}=S\left(T_{\mathcal{M}}^{\star}\right)$ and $\mathcal{M}=S^{0}\left(T_{\mathcal{M}}^{\star}\right)$ since $\mathcal{G}$ and $T_{\mathcal{M}}^{\star}$ are mutually coinitial. Thus $\Sigma_{\mathcal{M}}$ is a topological field.

Acknowledgements. We would like to thank the referee for several valuable remarks. 
714 M. Balcerzak, A. Bartoszewicz, J. Rzepecka and S. Wroński

\section{References}

[BBC] M. Balcerzak, A. Bartoszewicz, K. Ciesielski, On Marczewski-Burstin representations of certain algebras of sets, Real Anal. Exchange, 26 (20002001).

[BR] M. Balcerzak, J. Rzepecka, Marczewski sets in the Hashimoto topologies for measure and category, Acta Univ. Carolin. Math. Phys., 39 (1998), 93-97.

[Bre] J. Brendle, Strolling through paradise, Fund. Math. 148 (1995), 1-25.

[Br1] J. B. Brown, The Ramsey sets and related sigma algebras and ideals, the lecture during Special Session on Measure Theory and Descriptive Set Theory, Atlanta Meeting of the AMS, January 1988.

[Br2] J. B. Brown, The Ramsey sets and related sigma algebras and ideals, Fund. Math. 136 (1990), 179-185.

[BrCo] J. B. Brown, G. V. Cox, Classical theory of totally imperfect spaces, Real Anal. Exchange 7 (1982), 1-39.

[BET] J. B. Brown, H. Elalaoui-Talibi, Marczewski-Burstin-like characterizations of $\sigma$-algebras, ideals, and measurable functions, Colloq. Math., 82 (1999), 277-286.

[Bu] C. Burstin, Eigenschaften messbaren und nichtmessbaren Mengen, Wien Ber. 123 (1914), 1525-1551.

[CJ] K. Ciesielski, J. Jasinski, Topologies making a given ideal nowhere dense or meager, Topology Appl. 63 (1995), 277-298.

[CLO] K. Ciesielski, L. Larson, K. Ostaszewski, I-density continuous functions, Mem. Amer. Math. Soc. 107 (515) (1994).

[Co] P. Corazza, Ramsey sets, the Ramsey ideal, and other classes over $\mathbf{R}$, J. Symb. Logic 57 (1992), 1441-1468.

[F] F. J. Freniche, The number of nonisomorphic Boolean subalgebras of a power set, Proc. Amer. Math. Soc. 91 (1984), 199-201.

$[\mathrm{H}]$ H. Hashimoto, On the ${ }^{\star}$ topology and its application, Fund. Math. 91 (1976), 5-10.

[JH] D. Jankovič and T. R. Hamlett, New topologies from old via ideals, Amer. Math. Monthly, 97, No 4, April 1990, 295-310. 
[K] S. Kopelberg, Handbook of Boolean Algebras, vol.1, North Holland, 1989.

[Ku] K. Kuratowski, Topology, vol.1, Academic Press, New York, 1966.

[LMZ] J. Lukeš, J. Malý and L. Zajiček, Fine Topology Methods in Real Analysis and Potential Theory, Lecture Notes in Math. 1189, Springer, New York, 1986.

[LJW] E. Łazarow, R. A. Johnson, W. Wilczyński, Topologies related to sets having the Baire property, Demonstr. Math. 22 (1989), 179-191.

[Mi1] A. W. Miller, Special subsets of the real line, in Handbook of SetTheoretic Topology, North-Holland, Amsterdam-New York, 1984, 201-233.

[Mi2] A. W. Miller, Special sets of reals, Israel Math. Conf. Proc., 6 (1993), 415-431.

[Mo] J. C. Morgan II, Point Set Theory, Marcel Dekker, New York, 1990.

[O] J. C. Oxtoby, Measure and Category, Springer Verlag, New York 1971.

[Pa] J. Pawlikowski, Parametrized Ellentuck theorem, Topology Appl. 37 (1990), 65-73.

[PWW] W. Poreda, E. Wagner-Bojakowska, W. Wilczyński, A category analogue of the density topology, Fund. Math. 125 (1985), 167-173.

[R] P. Reardon, Ramsey, Lebesgue and Marczewski sets and the Baire property, Fund. Math. 149 (1996), 191-203.

[Sz] E. Szpilrajn (Marczewski), Sur une classe de fonctions de M. Sierpiński et la classe correspondante d'ensembles, Fund. Math. 24 (1935), 17-34.

[Wa] J. T. Walsh, Marczewski sets, measure and the Baire property, Fund. Math. 129 (1988), 83-89.

[W1] W. Wilczyński, A generalization of the density topology, Real Anal. Exchange 8 (1982-83), 16-20.

[W2] W. Wilczyński, A category analogue of the density topology, approximate continuity and the approximate derivative, Real Anal. Exchange 10 (198485), 241-265.

[Wr1] S. Wroński, On fields of sets with a nowhere dense boundary, Demonstr. Math. 12 (1979), 373-377.

[Wr2] S. Wroński, On proper subuniverses of a Boolean algebra, Acta Univ. Lodziensis, Folia Mathematica 9 (1997), 69-76. 
716 M. Balcerzak, A. Bartoszewicz, J. Rzepecka and S. Wroński 